\title{
Context change and retention interval can have additive, rather than interactive, effects after taste aversion extinction
}

\author{
JUAN M. ROSAS and MARK E. BOUTON \\ University of Vermont, Burlington, Vermont
}

\begin{abstract}
Spontaneous forgetting is often attributed to retrieval failure caused by natural changes in the background context that occur over time. However, some investigators have argued that the contextchange account of forgetting is paradoxical, because context-change effects themselves decrease over time. To resolve the paradox, we have suggested that organisms may merely forget the physical context as the temporal context in which it is embedded changes; this explanation accepts a fundamental similarity between time and physical context. The present experiment tested an implication of this analysis by examining the interaction between retention interval and context change in rats after a taste aversion was conditioned and then extinguished. Importantly, subjects tested at the longer (24-day) retention interval received reminder exposure to the physical contexts before testing. Under these conditions, retention interval and context change both caused relapse of the extinguished aversion (spontaneous recovery and renewal, respectively), and the strongest overall relapse was observed when the two treatments were combined. Such additivity (rather than interactivity) is consistent with a context-change account of forgetting and sets the stage for resolution of the contextforgetting paradox.
\end{abstract}

It is now common to suppose that spontaneous forgetting in humans and animals is caused by changes that naturally occur in the background context over time (e.g., Bouton, 1993; Estes, 1955; McGeoch, 1942; Mensink \& Raaijmakers, 1988; Spear, 1978; Tulving \& Thomson, 1973). Memory retrieval is assumed to be best when there is a match between the conditions present at learning and testing; mismatches that increase over time cause a failure to retrieve the target material. Consistent with the view that spontaneous forgetting is often due to retrieval failure, forgetting that occurs after a retention interval can often be undone by presenting appropriate retrieval cues (e.g., Gordon, 1981). Although this sort of result identifies retrieval failure as a mechanism of forgetting, to what extent is it really due to changes in contextual cues?

Bouton (1993) noted that retention intervals and context switches have surprisingly parallel effects on animal memory and that the parallel is consistent, in a preliminary way, with the possibility that they work through a similar mechanism. Conditioning phenomena that are disrupted by a retention interval are also disrupted by context change, whereas those that are not affected by time

This research was supported by National Science Foundation Grant IBN-9209454 to M.E.B. and by a grant from the Basque Government's Programa de Formación de Investigadores (Ref. BFI94.140) to J.M.R The authors thank Robert Boakes for his comments on the manuscript. Correspondence should be addressed to J. M. Rosas, Departamento de Psicologia, Universidad de Jaén, Paraje de las Lagunillas s/n, 23071Jaén, Spain, or to M. E. Bouton, Department of Psychology, University of Vermont, Burlington, VT 05405-0134 (e-mail: mbouton@zoo.uvm edu). are likewise not affected by context. For example, simple classical conditioning can be stable over long retention intervals (e.g., Hendersen, 1985) and over changes of physical context (e.g., Bouton \& King, 1983; Bouton \& Peck, 1989; Hall \& Honey, 1989). In contrast, latent inhibition (in which unreinforced preexposure to a Pavlovian signal interferes with its subsequent conditioning) is disrupted by both a retention interval (e.g., Aguado, Symonds, \& Hall, 1994) and a change of context (e.g., Channell \& Hall, 1983). Extinction (in which conditioned responding to a Pavlovian signal is reduced when it is presented alone repeatedly after conditioning) is also lost after both a retention interval (e.g., Brooks \& Bouton, 1993; Pavlov, 1927; Robbins, 1990) and after a context switch (e.g., Bouton \& King, 1983; Bouton \& Peck, 1989; Brooks \& Bouton, 1994). The latter two effects are better known as spontaneous recovery and the renewal effect, respectively. Both are reduced if a retrieval cue for extinction is presented just before the test (Brooks \& Bouton, 1993, 1994). Spontaneous recovery and renewal may thus result from a common mechanism - namely, a failure to retrieve extinction after a change in either the temporal or the physical context-further supporting the idea that time and physical context influence memory the same way.

Although these parallels are consistent with the idea that time and context can work through a common mechanism, the context-change account of forgetting has been challenged by an interesting paradox (Riccio, Rabinowitz, \& Axelrod, 1994; Riccio, Richardson, \& Ebner, 1984). Stimulus generalization may increase over longer retention intervals in both humans and animals (e.g., W. R. McAllister, D. E. McAllister, \& Franchina, 1965; Thomas, 
1981; Thomas \& Lopez, 1962; but see Hoffman, Selekman, \& Fleshler, 1966). Thus, humans and other animals appear to forget stimulus attributes, and they are therefore less likely to differentiate between similar stimuli over time. If contexts are subject to this sort of forgetting themselves, it would be paradoxical to claim that forgetting is caused by contextual change. How can contextual mismatch ever occur if contextual stimuli are judged to be increasingly similar over time?

The context-forgetting paradox predicts an interaction between context-switch and retention-interval effects, so that the size of a context-switch effect should decrease as retention interval increases. Although there is evidence consistent with this possibility (e.g., see Riccio et al., 1984 , for a review), we have noted that such a finding does not necessarily challenge the context-change account of forgetting (Rosas \& Bouton, 1997a). Physical contexts are naturally embedded within a superordinate context provided by the passage of time. Therefore, it would not be surprising to find that animals "forget" (or fail to retrieve) physical contexts as the temporal context changes, even if time and physical context affect memory through a common mechanism. This approach is consistent with a finding that has never been fully reconciled with the context-forgetting paradox. Specifically, generalization gradients that have flattened over time can be resharpened by presenting appropriate retrieval cues (e.g., Moye \& Thomas, 1982). For example, Zhou and Riccio (1994) found that a context-switch effect on passive avoidance was lost after a 7-day retention interval, but it could be reestablished by a brief exposure to the context shortly before testing. As Zhou and Riccio noted, such results suggest that generalization gradients flatten because of retrieval failure. But they did not address why such a failure should occur. We suggest that it comes about because of natural changes in the superordinate temporal context.

The purpose of the present experiment was to test an implication of this analysis. Bouton (1993, p. 91) noted that if the effects of physical context switches and retention intervals are based on the same underlying process, then physical context switches and retention intervals (temporal context switches) should have an additive effect. That is, a combination of physical context change and retention interval should cause greater forgetting than either treatment alone. Put casually, the effect of manipulating time and context together should create an even bigger context switch. However, to test that additivity, one needs to ensure that the physical context change is the same at the short and long retention intervals, a point that would be precluded if the animals generalized more between the contexts at the longer retention interval. One way to resharpen this generalization would be to remind the animals of the physical contexts before the test (Rosas \& Bouton, 1997a; Zhou \& Riccio, 1994). Therefore, in the present experiment, we used a context reminder procedure in order to provide an ade- quate test of the possibility that context change and retention intervals can have additive effects.

We examined this issue using extinction in a taste aversion conditioning paradigm. It is widely known that animals learn aversions to flavors that are associated with illness and that such aversions can be extinguished by repeated exposure to the flavor alone (e.g., Rosas \& Bouton, 1996). However, extinction does not destroy the original learning. As in other conditioning preparations, animals retain conditioning after extinction; performance after extinction is determined by which of two available memories (conditioning vs. extinction) is retrieved, with extinction being especially disrupted by context change (Bouton, 1991, 1993). We have recently reported both spontaneous recovery and renewal after the extinction of a conditioned taste aversion: When time elapses after extinction, or when the physical context is changed, a modest recovery of the aversion is observed (Rosas \& Bouton, 1996, 1997b). Because neither of these effects is especially large, the aversion extinction preparation leaves ample room on the response scale to observe additive effects if they exist. We therefore used taste aversion extinction to test the interaction between context and retention-interval effects. Animals tested at the long retention interval received reexposure to the context that might remind the animal of the context before testing began.

All rats received a pairing of a saccharin drink with illness in one apparatus (Context A). This created an aversion to the saccharin flavor. Half the rats then received extinction exposures to saccharin in the same context (Context A), and half received extinction in a different context (Context B). The apparatuses that provided the contexts were counterbalanced, and exposure to them was controlled by giving each rat alternating sessions in the contexts throughout the experiment. After the aversion was extinguished, all rats were tested with saccharin in Context A. We expected a renewed aversion in groups for which saccharin was returned from Context B to Context A (e.g., Rosas \& Bouton, 1997b). We factorially combined the context of extinction (A or B) with a retention interval of either 1 or 24 days between the last extinction trial and the test. Rats receiving the 24-day retention interval should show spontaneous recovery of the aversion (Rosas \& Bouton, 1996). The main question was whether the retention interval and context switch would combine additively to produce the strongest aversion in the group that received both manipulations.

\section{METHOD}

\section{Subjects}

The subjects were 32 female Wistar rats obtained from a commercial supplier (Charles River, Canada). They had previously served, while hungry, in an experiment in which tones were paired with food pellets in a different apparatus. The rats received ad-lib food and water for 3 weeks before beginning the present experiment. Throughout, they 
were housed individually in standard stainless steel cages. The experiment was conducted at the same times each day during the light portion of an 18:6-h light:dark cycle.

\section{Apparatus}

We used two counterbalanced sets of four boxes that have been used in previous research on the role of context in conditioning (e.g., Bouton \& Peck, 1989). The two sets differed in their location in the laboratory and on visual, tactile, and olfactory dimensions. Fluids were administered in the boxes through a stainless steel drinking spout. The experimental flavor was a $0.05 \%(\mathrm{w} / \mathrm{v})$ solution of sodium saccharin (Sigma Chemical Co., St. Louis). Illness was induced by a $2 \%$ bodyweight intraperitoneal injection of $0.15 \mathrm{M} \mathrm{LiCl}$.

\section{Procedure}

The rats first received 5 days of exposure to a drink schedule consisting of two daily 15 -min drinks of distilled water. The drinks were separated by $10 \mathrm{~h}$; this two-drinks-a-day schedule was maintained whenever deprivation was in force. On Days 1-3, water was presented in the colony room. On Days 4 and 5 , each rat received a drink in a box from each of the sets described earlier. Context sequence was counterbalanced. The rats were then assigned to groups in a way that matched them on water consumption and balanced the boxes and their order of presentation on these days.

We used the $2 \times 2$ factorial design summarized in Table 1 . To control for thirst level on the final test, we arranged the procedure so that all testing occurred on Day 36 of the experiment. This meant that the animals that received testing after the longer (24-day) retention interval received conditioning on Day 6 and extinction on Days 8-12, whereas the rats that received the shorter (1-day) retention interval received conditioning on Day 29 and extinction on Days 31-35. (Days 7 and 30 involved exposure only to water.) The procedure guaranteed that the different groups differed in when and/or where they experienced conditioning and extinction with the saccharin, but not in their experience with the two contexts or the deprivation schedule.

On the appropriate conditioning day, each rat received access to saccharin for $15 \mathrm{~min}$ in Context $\mathrm{A}$. $\mathrm{LiCl}$ was then injected within $10 \mathrm{~min}$ The rats were returned to and remained in the context for $15 \mathrm{~min}$ after the injection. They received equivalent exposure to drinking water in Context B during the other drink period of the same day. (Boxes were cleaned between rats to avoid any possible odor produced as an after-
Table 1

Experimental Design

\begin{tabular}{|c|c|c|c|c|c|}
\hline Group & $\begin{array}{l}\text { Conditioning } \\
\text { (Day 6) }\end{array}$ & $\begin{array}{l}\text { Extinction } \\
\text { (Days 8-12) }\end{array}$ & $\begin{array}{l}\text { Conditioning } \\
\text { (Day 29) }\end{array}$ & $\begin{array}{c}\text { Extinction } \\
\text { (Days } 31-35)\end{array}$ & $\begin{array}{c}\text { Test } \\
\text { (Day 36) }\end{array}$ \\
\hline A. & $\mathrm{A} / \mathrm{B}$ & & & & \\
\hline$B-$ & & & & & \\
\hline & & & & & \\
\hline B-24 & $\mathrm{AS}+/ \mathrm{B}$ & $\mathrm{A} / \mathrm{BS}-$ & $A / B$ & $\mathrm{~A} / \mathrm{B}$ & $\mathrm{AS}-/ \mathrm{B}$ \\
\hline
\end{tabular}

Note- $A$ and $B$, two different contexts; $S$, saccharin; $S+$, saccharin paired with $\mathrm{LiCl}$ injection; $\mathrm{S}-$, saccharin without injection.

effect of the injection.) A recovery day followed in which the rats received water at the usual times in the home cage. On each of the next 5 days, the rats received saccharin during one of their daily $15-\mathrm{min}$ drinks. The animals receiving these extinction trials in Context A received saccharin in $A$ and water in $B$; the animals receiving extinction in Context $B$ received saccharin in $B$ and water in $A$. As usual, because of the counterbalancing of box and box sequence, half the rats receiving each treatment received saccharin as the first drink of the day and half received it as the second drink.

As shown in Table 1, in order to control exposure to the contexts throughout the experiment, the rats that did not receive conditioning or extinction treatments on a given day simply received water in the corresponding contexts at the corresponding times. On Days 13-23, all rats received ad-lib tap water in the home cage.

The test took place on Day 36. For the rats tested at the long retention interval, this was 24 days after the last extinction trial; for the rats tested at the short interval, this was 1 day after the last extinction trial. At this time, all subjects received access to saccharin for $15 \mathrm{~min}$ in Context $A$ and water in Context $B$ at the appropriate times. Note again that the method controlled the pattern of deprivation and box exposure throughout the experiment. Group designations are defined in Table 1.

The data of 2 rats ( 1 in Group A-1 and 1 in Group B-1) were discarded because of experimenter error (incorrect exposure to water or saccharin in a particular context). Data of 2 other rats ( 1 in Group A24 and 1 in Group B-24) were also dropped because their aversion never extinguished (they did not consume a measurable amount of saccharin on any extinction trial).
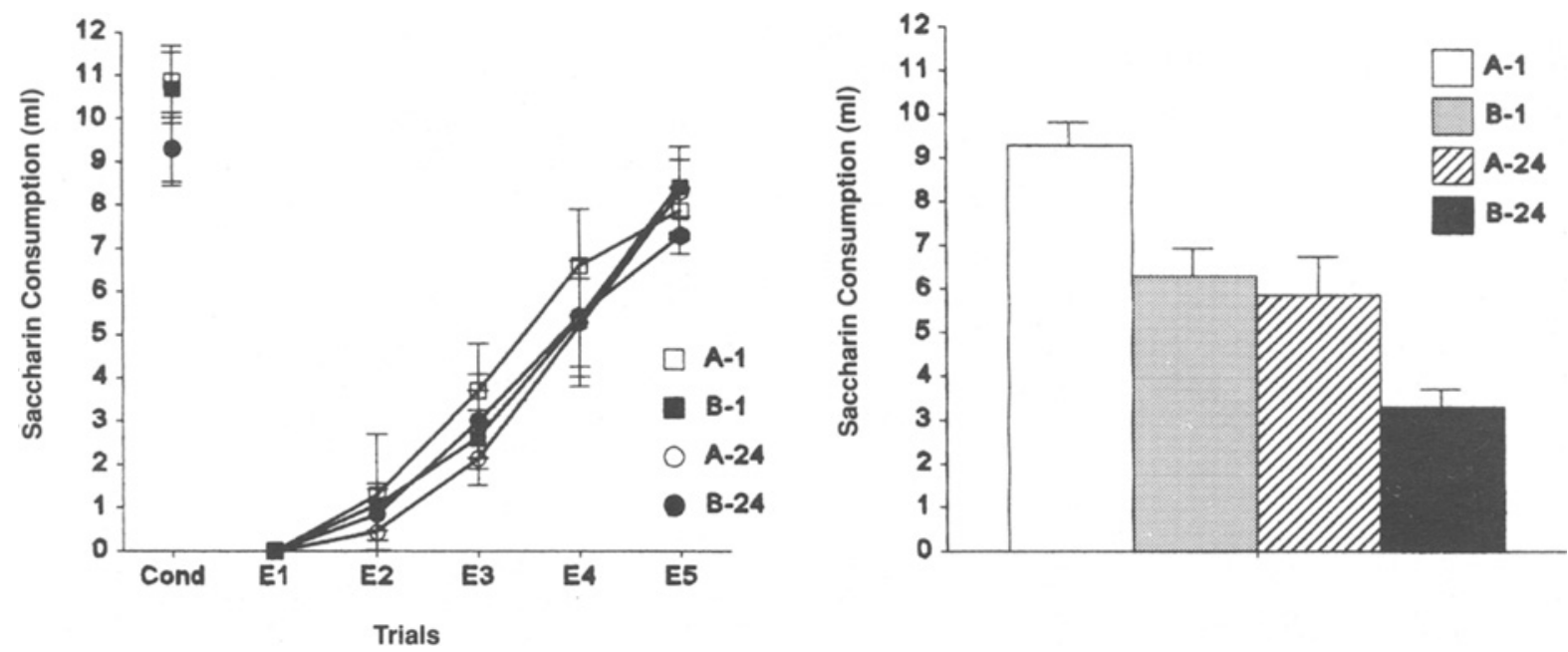

Figure 1. Mean saccharin consumption on the conditioning day and the 5 days of extinction (left panel) and on the day of testing (right panel) for Groups A-1, B-1, A-24, and B-24. Conditioning and testing took place in Context A for all subjects. Groups B-1 and B-24 received extinction in a context different from the conditioning and testing context; Groups A-1 and A-24 received conditioning, extinction, and testing in the same context (A). Groups A-24 and B-24 finished extinction 24 days before the test day; Groups A-1 and B-1 finished extinction the day before testing. 


\section{RESULTS}

The left panel of Figure 1 shows the groups' saccharin intake on each of the conditioning and extinction trials. There were no differences in consumption on the conditioning trial, as revealed by a $2 \times 2$ (retention interval $\times$ extinction context) analysis of variance (ANOVA) [largest $F(1,24)=3.52, p>.07]$. Saccharin consumption decreased precipitously after conditioning and then gradually increased during extinction. As is usually the case in our laboratory, extinction proceeded at the same rate regardless of whether it occurred in the context of conditioning (Context A) or in the alternate context (Context B). The 1-day and 24-day groups also did not differ. A retention interval $\times$ extinction context $\times$ extinction trial ANOVA revealed a significant main effect of trial $[F(1,24)$ $=113.24, p<.0001]$, but no other main effect or interaction [largest $F(4,96)=1.24, p s>.29]$. Thus, neither the context of extinction nor the timing of the conditioning and extinction phases had a measurable impact on the aversion.

The test results are presented in the right panel of Figure 1. Both the return to Context $\mathrm{A}$ after extinction in Context B (Group B-1) and the 24-day retention interval (Group A-24) produced a moderate decrease in saccharin consumption, relative to the group that did not receive either the context switch or retention interval between extinction and testing (Group A-1). Most importantly, the decrease in consumption was even greater in the group that received both the context switch and the retention interval (Group B-24). A retention interval $\times$ context ANOVA found significant main effects of retention interval $[F(1,24)=25.10, p<.0001]$ and context switch $[F(1,24)=18.86, p<.001]$ and no interaction between these two factors $(F<1)$. Planned comparisons revealed that Groups B-1 (context switch) and A-24 (retention interval) each showed less consumption than did Group A-1 (extinction control) $[F \mathrm{~s}(1,24) \geq 10.93, p \mathrm{~s}<.01]$. Most importantly, there was less saccharin consumption in Group B-24 (context switch and retention interval combined) than in either Group B-1 or Group A-24 $[F \mathrm{~s}(1,24)$ $\geq 8.03, p \mathrm{~s}<.01]$. These findings are entirely consistent with the view that time and physical context change can have additive, rather than interactive, effects.

\section{DISCUSSION}

The fact that the taste aversion recovered as a result of the contextswitch and retention-interval manipulations is consistent with previous research on taste aversion learning (Rosas \& Bouton, 1996, 1997b) and other types of conditioning (e.g., Brooks \& Bouton, 1993, 1994). Extinction performance is relatively specific to the time and place in which it is learned. The fact that changing the context after conditioning did not weaken the conditioned response (taste aversion) is also consistent with research using a number of other conditioning methods (e.g., Bouton \& King, 1983; Bouton \& Peck, 1989; Brooks \& Bouton, 1994; Hall \& Honey, 1989). It is interesting that the rules governing extinction and relapse of a taste aversion seem so similar to those governing other types of associative learning; taste aversions have some- times been thought to follow special laws (e.g., Rozin \& Kalat, 1971). The overall pattern of the present results is consistent with previous research suggesting that retrieval of extinction is especially specific to its physical and temporal context (Bouton, 1993).

The major finding, however, is that the context-switch and retentioninterval manipulations had an even greater effect when they were combined. The group that received both the context switch and the long retention interval after extinction (Group B-24) showed a stronger recovery of the aversion than did the groups that received either manipulation alone (Groups B-1 and A-24). There were clear effects of the contextswitch and retention-interval factors, with no interaction between them (cf. Riccio et al., 1984). This resuit contrasts with previous reports suggesting that context-switch effects may diminish over time (c.g., GisquetVerrier \& Alexinsky, 1986; Kissinger \& Riccio, 1995; W. R. McAllister \& D. E. McAllister, 1963; Perkins \& Weyant, 1958; see Riccio et al., 1994, and Riccio et al., 1984, for reviews). One variable that makes our experiment different from most is that we equated exposure to the two contexts throughout the experiment. Previous experiments have often switched to a completely novel context, introducing the possibility that competing exploratory behaviors, rather than true memory loss, might have reduced performance (Devenport, 1989; Gisquet-Verrier \& Alexinsky, 1986). A second variable that makes this experiment different is that the rats tested after the long retention interval also received reexposure to the context for several days just before the test day. If contextual attributes are indeed forgotten over time, this reexposure would have reminded the animals of the two contexts and thus equated the context-switch manipulation performed at the long and short retention intervals. Without such a reminder, if the animal does forget contextual attributes, then a context switch at a longer retention interval cannot be considered comparable to a switch at a shorter interval, and, thus, the additivity hypothesis investigated here would not be adequately tested.

These results are consistent with the findings of related experiments in our laboratory on latent inhibition in appetitive conditioning (Rosas \& Bouton, 1997a). In those experiments, we gave rats tone-alone presentations before pairing the tone with food; as described above, preexposure to the tone interfered with conditioning that resulted from the tone-food pairings. A context switch or retention interval between preexposure and conditioning have both been shown to reduce latent inhibition, perhaps by causing a failure to retrieve the preexposure experience (see Hall, 1991, for a review). When we first tested both manipulations together, the size of the context-switch effect on latent inhibition was reduced after a long retention interval, as the contextforgetting paradox predicts (Rosas \& Bouton, 1997a, Experiment 1). However, when the rats were reexposed to the contexts before testing, the context-switch effect returned. In this case, the time- and contextswitch effects were additive, so that the greatest loss of latent inhibition was evident in a group that received both the retention interval and the context switch (Rosas \& Bouton, 1997a, Experiment 2). We suggested that reexposure to the context improved memory for the contexts during the test and revealed an additivity between time and context that is consistent with a common mechanism.

These converging demonstrations of additivity in two different preparations are consistent with, although alone they do not force, the conclusion that time and context effects may be based on a common mechanism. That is, retention intervals may cause changes in memory performance at least in part because they are correlated with natural changes in background contextual cues. The additivity result is but one of a number of results consistent with this idea. As Bouton (1993) noted, and as we reviewed above, there is a broad parallel between the effects of retention interval and context switches in the literature. Those phenomena (e.g., extinction and latent inhibition) that are affected by time are usually also affected by a change in the context, whereas those that are resistant to the effects of retention intervals (i.e., simple conditioning) are also relatively resistant to the effect of physical context change (see Bouton, 1993, for a review). As an additional line of evidence, Brooks and Bouton $(1993,1994)$ found that renewal and spontaneous recovery effects, analogous to the ones observed here in Groups B-1 and A-24, are both attenuated by presenting a retrieval cue for extinction at the time of testing. Taken together, the results are 
consistent with the idea that time and context switches work through a common mechanism.

The present results are also compatible with our resolution of the context-forgetting paradox (Rosas $\&$ Bouton, 1997a). The fact that animals can forget contextual attributes over time (e.g., Riccio et al., 1984) does not necessarily challenge the context-change account of forgetting. Physical contexts are necessarily embedded in a larger temporal context, so that retention intervals can be expected to affect the memory of physical contexts the same way they affect the memory of other embedded information. Such forgetting can cause a weaker context effect at long retention intervals under some circumstances. But in procedures, like the present one, that ensure adequate memory for the physical contexts during the test, context-switch and retention-interval effects can be shown to be additive rather than interactive. This suggests that even the diminishing effects over time of a change in the physical context might be caused by changes in the temporal context. Thus, the widely held but rarely tested idea that spontaneous forgetting is due in part to contextual change may remain, for the time being, secure.

\section{REFERENCES}

Aguado, L., Symonds, M., \& HaLl, G. (1994). Interval between preexposure and test determines the magnitude of latent inhibition: Implications for an interference account. Animal Learning \& Behavior, 22, 188-194

Bouton, M. E. (1991). Context and retrieval in extinction and in other examples of interference in simple associative learning. In L. W. Dachowski \& C. F. Flaherty (Eds.), Current topics in animal learning: Brain, emotion, and cognition (pp. 25-53). Hillsdale, NJ: Erlbaum.

Bouton, M. E. (1993). Context, time, and memory retrieval in the interference paradigms of Pavlovian learning. Psychological Bulletin, 114, 80-99.

Bouton, M. E., \& KING, D. A. (1983). Contextual control of the extinction of conditioned fear: Tests for the associative value of the context. Journal of Experimental Psychology: Animal Behavior Processes, 9, 248-265.

Bouton, M. E., \& PeCK, C. A. (1989). Context effects on conditioning, extinction, and reinstatement in an appetitive conditioning preparation. Animal Learning \& Behavior, 17, 188-198.

Brooks, D. C., \& Bouton, M. E. (1993). A retrieval cue for extinction attenuates spontaneous recovery. Journal of Experimental Psychology: Animal Behavior Processes, 19, 77-89.

Brooks, D. C., \& Bouton, M. E. (1994). A retrieval cue for extinction attenuates response recovery (renewal) caused by a return to the conditioning context. Journal of Experimental Psychology: Animal Behavior Processes, 20, 366-379.

Channell, S., \& Hall, G. (1983). Contextual effects in latent inhibition with an appetitive conditioning procedure. Animal Learning \& Behavior, 11, 67-74

DEVENPORT, L. (1989). Sampling behavior and contextual change Learning \& Motivation, 20, 97-114.

EsTES, W. K. (1955). Statistical theory of spontaneous recovery and regression. Psychological Review, 62, 145-154.

Gisquet-VerRier, P., \& ALEXINSKY, T. (1986). Does contextual change determine long-term forgetting? Animal Learning \& Behavior, 14 349-358.

GORDON, W. C. (1981). Mechanisms of cue-induced retention enhancement. In N. E. Spear \& R. R. Miller (Eds.), Information processing in animals: Memory mechanisms (pp. 319-340). Hillsdale, NJ: Erlbaum.

HALL, G. (1991). Perceptual and associative learning. Oxford: Oxford University Press, Clarendon Press.

HaLL, G., \& HoNEY, R. C. (1989). Contextual effects in conditioning, latent inhibition, and habituation: Associative and retrieval functions of contextual cues. Journal of Experimental Psychology: Animal Behavior Processes, 15, 232-241.

HENDERSEN, R. W. (1985). Fearful memories: The motivational significance of forgetting. In F. R. Brush \& J. B. Overmier (Eds.), Affect conditioning, and cognition: Essays on the determinants of behavior (pp. 43-54). Hillsdale, NJ: Erlbaum.

Hofrman, H. S., Selekman, W., \& Fleshler, M. (1966). Stimulus aspects of aversive controls: Long term effects of suppression procedures. Journal of the Experimental Analysis of Behavior, 9, 659-662.

Kissinger, S. C., \& Riccio, D. C. (1995). Stimulus conditions influencing the development of tolerance to repeated cold exposure in rats. Animal Learning \& Behavior, 23, 9-16.

MCAllister, W. R., \& MCAlLister, D. E. (1963). Increase over time in the stimulus generalization of acquired fear. Journal of Experimental Psychology, 65, 576-582.

McAllister, W. R., McAllister, D. E., \& Franchina, J. J. (1965). Dependence of equality judgments upon the temporal interval between stimulus presentations. Journal of Experimental Psychology, 70, 602-605

McGeoch, J. A. (1942). The psychology of human learning: An introduction. New York: Longmans, Green.

Mensink, G., \& RaAijmakers, J. G. W. (1988). A model for interference and forgetting. Psychological Review, 95, 434-455.

Moye, T. B., \& Thomas, D. R. (1982). Effects of memory reactivation treatments on postdiscrimination generalizaton performance in $\mathrm{pi}$ geons. Animal Learning \& Behavior, 10, 159-166.

Pavlov, I. P. (1927). Conditioned reflexes (G. V. Anrep, Trans.). London: Oxford University Press.

Perkins, C. C., JR., \& Weyant, R. G. (1958). Intertrial interval between training and test trials as determiner of the slope of generalization gradients. Journal of Comparative \& Physiological Psychology, 51, 596-600.

Riccio, D. C., Rabinowitz, V. C., \& AXelrod, S. (1994). Memory: When less is more. American Psychologist, 49, 917-926.

Riccio, D. C., Richardson, R., \& EBNer, D. L. (1984). Memory retrieval deficits based upon altered contextual cues: A paradox. Psychological Bulletin, 96, 152-165.

RoBBINS, S. J. (1990). Mechanisms underlying spontaneous recovery in autoshaping. Journal of Experimental Psychology: Animal Behavior Processes, 16, 235-249.

Rosas, J. M., \& Bouton, M. E. (1996). Spontaneous recovery after extinction of a conditioned taste aversion. Animal Learning \& Behavior, 24, 341-348.

Rosas, J. M., \& Bouton, M. E. (1997a). Additivity of the effects of retention interval and context change on latent inhibition: Toward resolution of the context forgetting paradox. Journal of Experimental Psychology: Animal Behavior Processes, 23, 283-294.

Rosas, J. M., \& Bouton, M. E. (1997b). Renewal of conditioned taste aversion upon return to the conditioning context after extinction in another one. Learning \& Motivation, 28, 216-229.

Rozin, P., \& Kalat, J. W. (1971). Specific hungers and poison avoidance as adaptive specializations of learning. Psychological Review, $\mathbf{7 8 ,}, 459-486$.

SPEAR, N. E. (1978). The processing of memories: Forgetting and retention. Hillsdale, NJ: Erlbaum.

Thomas, D. R. (1981). Studies of long-term memory in the pigeon. In N. E. Spear \& R. R. Miller (Eds.), Information processing in animals: Memory mechanisms (pp. 257-290). Hillsdale, NJ: Erlbaum. Thomas, D. R., \& LOPEZ, L. J. (1962). The effect of delayed testing on generalization slope. Journal of Comparative \& Physiological Psychology, 44, 541-544.

Tulving, E., \& Thomson, D. M. (1973). Encoding specificity and retrieval processes in episodic memory. Psychological Review, 80, 359-380.

Zhou, Y. L., \& Riccio, D. C. (1994). Pretest cuing can alleviate the forgetting of contextual stimulus attributes. Learning \& Motivation, 12, 233-244

(Manuscript received January 22, 1997; revision accepted for publication August 6, 1997.) 